\title{
Wallerian degeneration of bilateral cerebral peduncles after acute carbon monoxide poisoning
}

\author{
Sui-yi $\mathrm{Xu}^{1,2+}$, Chang-xin Li ${ }^{1 \dagger}$, Le-yi Li ${ }^{3}$, Yu Song ${ }^{3}$ and Yi Sui ${ }^{4^{*}}$
}

\begin{abstract}
Background: Cases of Wallerian degeneration of bilateral cerebral peduncles after acute carbon monoxide poisoning have not yet been reported. To date, most of the delayed encephalopathy after acute carbon monoxide poisoning (DEACMP) lesions captured in magnetic resonance imaging (MRI) has been located in the subcortical white matter and basal ganglia. Here we report two cases of DEACMP with abnormalities in the bilateral cerebral peduncles. The etiology of abnormalities, which were strictly confined to the bilateral cerebral peduncles, was Wallerian degeneration secondary to upstream nerve axonal damage, making this the first report on such bilateral cerebral peduncle abnormalities after DEACMP.

Case presentation: In this report, we present two cases of DEACMP with abnormal signals in the bilateral cerebral peduncles captured during brain MRIs. Case 1 was of a 68-year-old man who presented with paroxysmal disturbance of the consciousness, left limb weakness for 16 days, and lagging responses for 2 days. Case 2 was of a 55-year-old man who was unconscious for $6 \mathrm{~h}$. In addition to the above mentioned characteristics on the brain MRIs, the electroencephalography of case 1 indicated that his forehead scans had a mixture of wide sharp, sharp, and three-phase waves. Brain diffusion tensor imaging of case 2 further proved that the bilateral cerebral anomalies represented Wallerian degeneration secondary to upstream axonal damage. After the definitive diagnosis, the patients returned to the local hospital for hyperbaric oxygen therapy.

Conclusions: Wallerian degeneration of the bilateral cerebral peduncles after acute carbon monoxide poisoning has never been reported before. The abnormal signals in the bilateral cerebral peduncles captured during brain MRIs indicated Wallerian degeneration secondary to upstream axonal damage; thus, these two cases may further our understanding of DEACMP imaging.
\end{abstract}

Keywords: Wallerian degeneration, Cerebral peduncles, Carbon monoxide, Delayed encephalopathy

\footnotetext{
* Correspondence: neurosy@sina.com

${ }^{\dagger}$ Chang-xin Li and Sui-yi Xu contributed equally to this work.

${ }^{4}$ Department of Neurology and Neuroscience, Shenyang First People's

Hospital, Shenyang Brain Hospital, Shenyang Brain Institute, Shenyang Medical College, Shenyang, China

Full list of author information is available at the end of the article
}

(c) The Author(s). 2020 Open Access This article is licensed under a Creative Commons Attribution 4.0 International License, which permits use, sharing, adaptation, distribution and reproduction in any medium or format, as long as you give appropriate credit to the original author(s) and the source, provide a link to the Creative Commons licence, and indicate if changes were made. The images or other third party material in this article are included in the article's Creative Commons licence, unless indicated otherwise in a credit line to the material. If material is not included in the article's Creative Commons licence and your intended use is not permitted by statutory regulation or exceeds the permitted use, you will need to obtain permission directly from the copyright holder. To view a copy of this licence, visit http://creativecommons.org/licenses/by/4.0/ The Creative Commons Public Domain Dedication waiver (http://creativecommons.org/publicdomain/zero/1.0/) applies to the data made available in this article, unless otherwise stated in a credit line to the data. 


\section{Background}

Carbon monoxide (CO) is a colorless and odorless gas. $\mathrm{CO}$ poisoning is often caused by improper use of coal stoves for heating and is the cause of the suicide epidemic by charcoal burning in Southeast Asia in recent years [1]. CO poisoning has the dual effect of hypoxia and $\mathrm{CO}$ poisoning. Hypoxia itself can cause encephalopathy [2], and neurological damage caused by $\mathrm{CO}$ can lead to delayed encephalopathy after acute carbon monoxide poisoning (DEACMP). The diagnostic criterion for DEACMP [3] includes any of the following clinical abnormalities observed 2-60 days after the recovery of the consciousness disorder caused by acute $\mathrm{CO}$ poisoning: 1) disturbances of mental state and/or consciousness (such as dementia or delirium), 2) extrapyramidal lesions (such as Parkinson's syndrome), and 3) pyramidal damage and focal cortical dysfunctions.

Magnetic resonance imaging (MRI) suggests that most DEACMP lesions are located in the subcortical white matter and basal ganglia. Clinical manifestations include cognitive impairment, dyskinesia, forced crying, forced laughter, chorea, and Parkinson's syndrome $[4,5]$. However, bilateral cerebral peduncle anomalies captured on MRIs have not been reported. Herein we report two cases of DEACMP with Wallerian degeneration of the bilateral cerebral peduncles from different medical centers in China.

\section{Case presentation \\ Case 1}

The patient was a 68-year-old man who heated his home by burning wood or coal. Sixteen days prior, relatives found the patient lying on the ground in his room, unconscious, accompanied by vomit and incontinence. At the time of discovery, his relatives found that the coal stove was extinguished. He was alert during the brain computed tomography (CT) scan and complained of left limb weakness. The patient was diagnosed with a cerebral infarction and treated with antiplatelets and statin. Two days prior, the patient lagged in responses and would not eat on his own, and was transferred to our hospital. The patient had a prior medical history of prostatic hyperplasia (2 years ago), which had not been treated. He was a drinker and had been consuming alcohol ( $50 \mathrm{ml}$, twice a day) for more than 40 years.

On admission, his blood pressure was $140 / 90 \mathrm{mmHg}$. He was found to be lagging in responses and had memory deterioration. His left nasolabial groove was shallow. There were no other positive signs of nervous system impairment. On the third day after hospitalization, the patient's nervous system symptoms worsened. Physical examination revealed that he had difficulty in understanding, expression, memory, character, and spatial orientation. His tongue was slightly to the right. His right proximal lower extremity muscle strength was grade $4+$, and his distal muscle strength was grade 3.

No obvious abnormalities were revealed during his emergency brain CT and electrocardiography. His serological analysis data, such as arterial blood carboxyhemoglobin, blood sugar, glycosylated hemoglobin, electrolytes, blood cholesterol, coagulation function, myocardial enzymes, myohemoglobin, troponin, hepatic function, renal function, rheumatism series findings, thyroid function, folacin, vitamin B12, treponema pallidum antibody, hepatitis antigens and antibodies, and human immunodeficiency virus antibody, were found to be within normal limits. Certain laboratory indicators including thyroid stimulating hormone, blood sedimentation, and prostate specific antigens, were slightly elevated, but not specific. Cerebrospinal fluid findings which included sugar, chloride, protein, cell count, acid-fast staining results, ink staining results, autoimmune encephalitis, and paraneoplastic series findings, were normal.

His MRI showed an abnormal symmetrical signal in the bilateral basal ganglia, which was considered to be indicative of DEACMP (Fig. 1a). Of note, the bilateral cerebral peduncles also showed abnormal symmetrical signals (Fig. 1b-c). The patient's EEG indicated that Fp1, Fp2, F4, F8 leads in the frontal lobe area had a mixture of wide sharp, sharp, and three-phase waves (Fig. 2). Based on the above clinical manifestations and examination results, the patient was diagnosed with DEACMP. We recommend that the patient be treated with hyperbaric oxygen therapy (HBO). The relatives decided to return the patient to the local hospital to continue rehabilitation. At hospital discharge, the patient had blurred consciousness, indifferent expression, poor understanding, poor memory, poor orientation of characters and space, slow response, and low speech. His tongue was slightly to the right. Proximity muscle strength of right lower limb was grade $4+$, and distal muscle strength was grade 3 -.

\section{Case 2}

The patient was a 55-year-old man who was unconsciousness for $6 \mathrm{~h}$. The patient lived in the countryside. He heated his home by burning coal. He was found unconscious in a room with the smell of soot. The patient could only answer to simple questions and was brought to our hospital by an ambulance. The patient had a prior medical history of hypertension, gastric ulcers, and diabetes for more than 10 years. He had a suspected history of cerebral infarction for half a year, and his left side limbs were less flexible.

On admission, his blood glucose was $11 \mathrm{mmol} / \mathrm{L}$, and his blood pressure was 129/84 mmHg. He remained unconscious during the physical examination. His limbs moved voluntarily. His muscle tone was normal, and he 


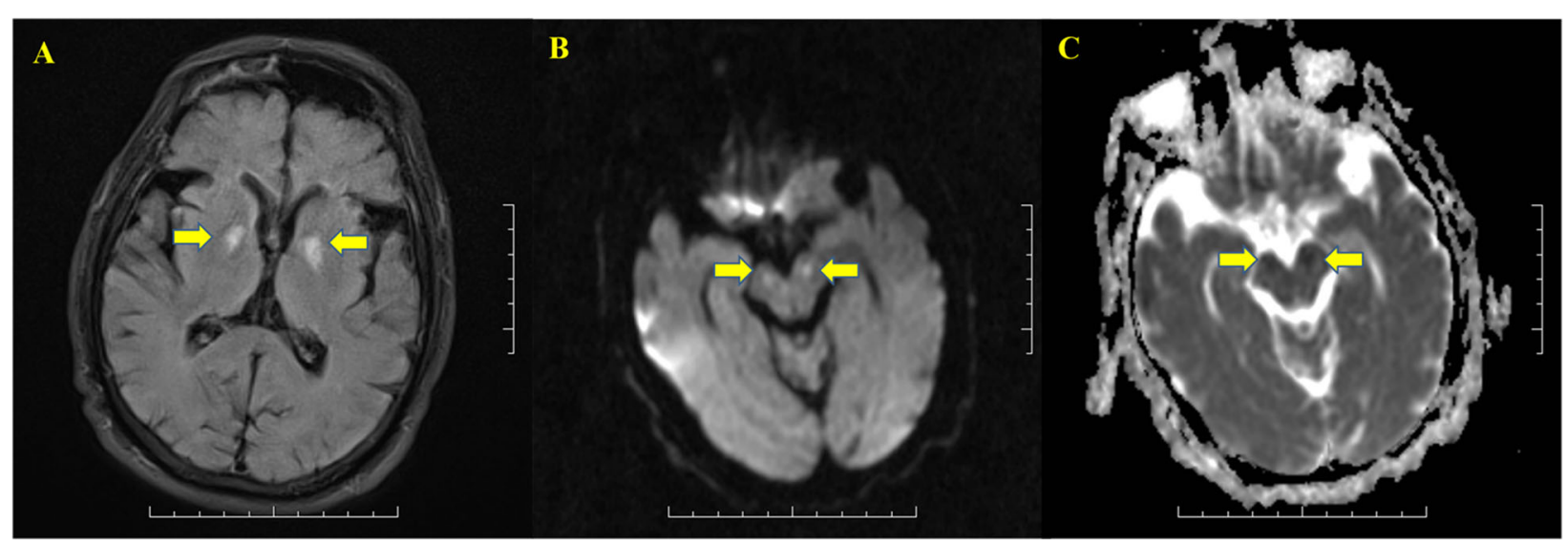

Fig. 1 A bilateral symmetrical anomaly signal of the basal ganglia was captured in fluid attenuated inversion recovery images (a). Bilateral cerebral peduncles showed symmetrical high signals in DWI (b), but low signals in ADC

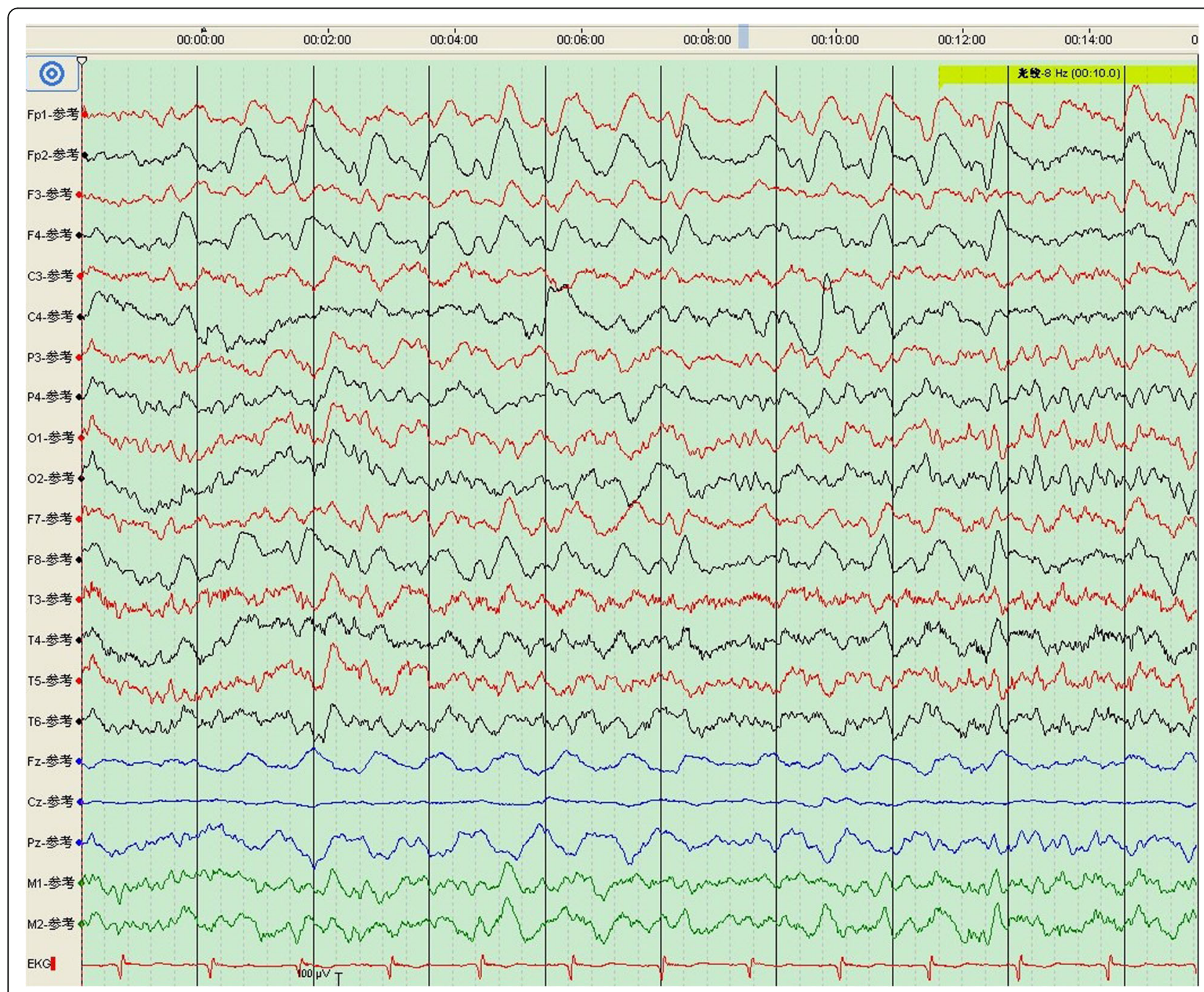

Fig. 2 The EEG indicated a diffuse distribution of Delta waves. The Fp1, Fp2, F4 in the frontal lobe area had a mixture of wide sharp, sharp, and three-phase waves. No clinical events occurred during the monitoring process 
had no muscle atrophy. Bilateral tendon reflexes could be symmetrically elicited. The Babinski sign and meningeal irritation were negative. His serological parameters which included blood cell findings, electrolytes, B type natriuretic peptide precursors, procalcitonin, liver enzymes, renal function, coagulation function, and Ddimer, were found to be within normal limits. The patient's glycated hemoglobin and homocysteine levels were above normal.

His brain CT scan showed no obvious abnormalities. The patient was treated with $\mathrm{HBO}$, antiplatelet, statin, and hypoglycemic agents. On the second day after admission, the patient was conscious. Interestingly, similar to case 1, the bilateral cerebral peduncles showed high symmetrical signals on brain diffusion-weighed imaging (DWI) (Fig. 3a), and low signals at corresponding areas in apparent dispersion coefficient (ADC) sequences (Fig. 3b). Diffusion tensor imaging (DTI) confirmed Wallerian degeneration secondary to nerve fiber bundle injury (Fig. 3c). The patient was diagnosed with DEACMP and treated with $\mathrm{HBO}$ until discharge from the hospital after 4 weeks. Moreover, the patient was treated with antiplatelet, statin and hypoglycemic agents because of previous stroke and diabetes history (Fig. 4). At hospital discharge, the patient was conscious. His speech was slightly slurred. The muscle strength was grade 4 with increased muscle tone.

\section{Discussion and conclusions}

In this report, we present two cases of abnormal signals in bilateral cerebral peduncles after carbon monoxide poisoning (Fig. 4). Both cases manifested as a disturbance of consciousness, which meet the diagnostic criteria of DEACMP as previously described [3]. However, the differential diagnosis of abnormal signals in bilateral cerebral peduncles in MRI should be identified (Table 1) [6-8]. Especially it needs to be distinguished from ischemic stroke when high signals are present on DWI while low signals occur in ADC. Of note, DWI/ADC lesions do not necessarily mean the occurrence of Wallerian degeneration. The restricted diffusion of white matter lesion might indicate slow and progressive development of cellular edema as a result of neuronal death and delayed demyelination in DEACMP [9]. In the case two patient, DTI was used to confirm that the abnormal signals of bilateral cerebral peduncles were Wallerian degeneration or just the sign of DEACMP.

Although basal ganglion necrosis is often mentioned in the literature for carbon monoxide poisoning, the incidence of globus pallidus lesions is significantly lower

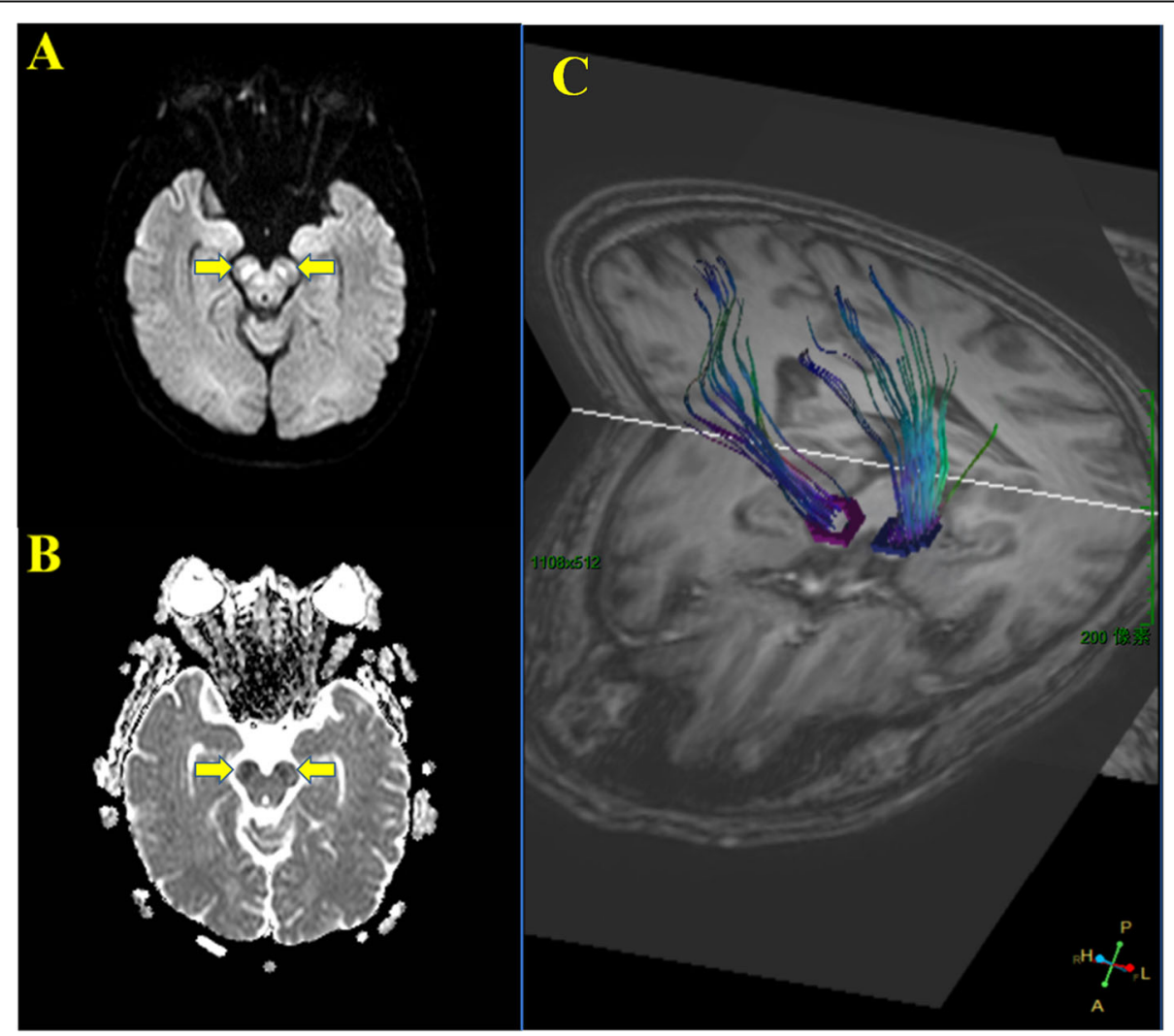

Fig. 3 Similar to case 1, bilateral cerebral peduncles showed symmetrical high signals in DWI (a), but low signals in ADC (b). Wallerian degeneration of bilateral cerebral peduncles secondary to corticospinal tract injury was confirmed by DTI. 


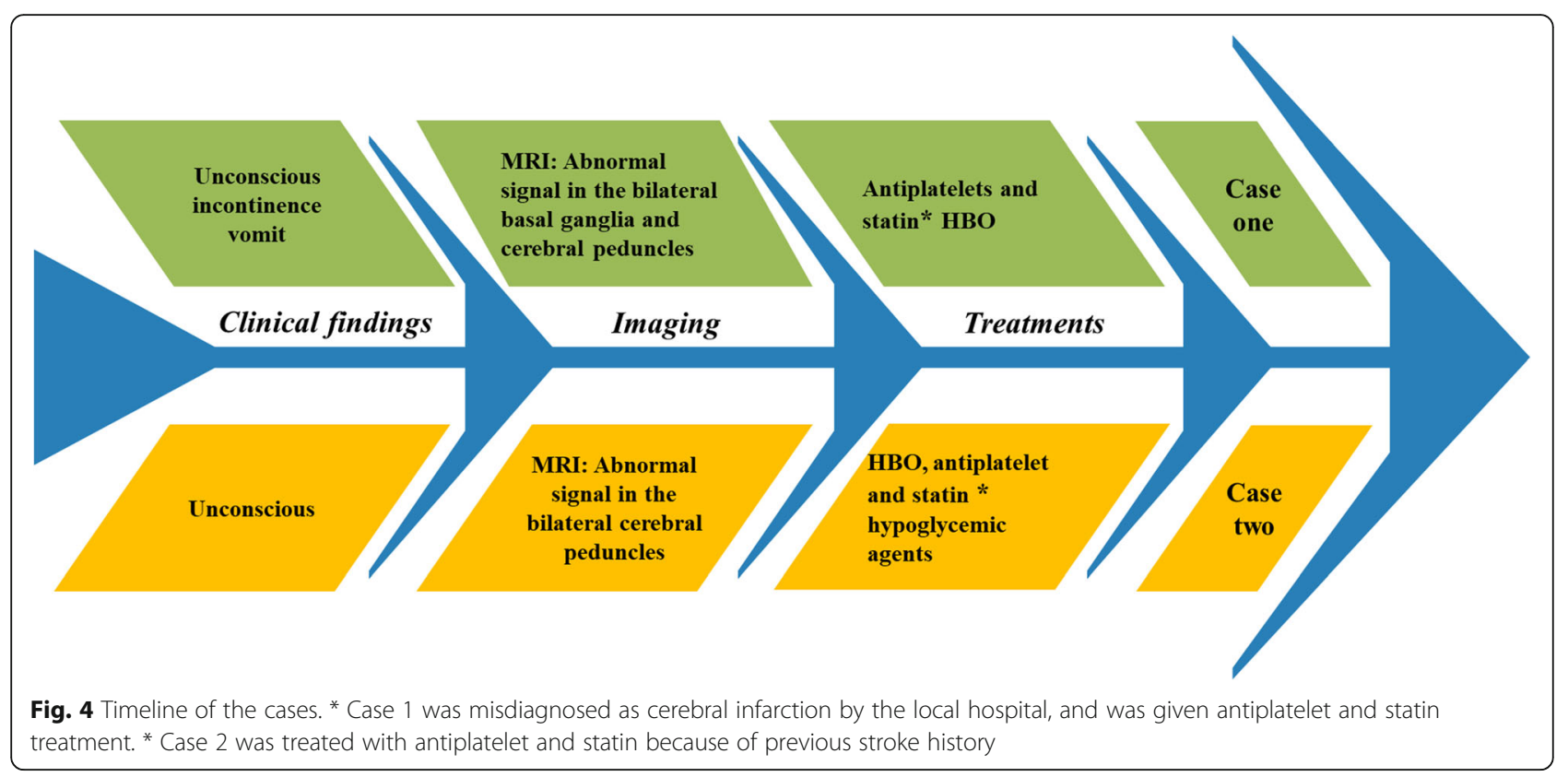

than that of white matter lesions [1]. As early as 1982 [10], some scholars found that early white matter lesions in carbon monoxide poisoning were neither true demyelination nor selective involvement of oligodendrocytes, but rather axonal damage with glial changes followed by Wallerian degeneration. Glial activation may play an important role in DEACMP [11], with serum S100B or glial fibrillary acidic protein levels being related to the prognosis. A recent study reported [12] that demyelination by $\mathrm{CO}$ is more severe than that by simple hypoxia, and the former lasts longer.

At present, there are few EEG reports for DEACMP. In Case 1 reported here, the patient's EEG indicated that Fp1, Fp2, F4, F8 leads in the frontal lobe area had a mixture of wide sharp, sharp, and three-phase waves which implied forehead cortical dysfunction. In a case report regarding generalized chorea caused by DEACMP [5], the patient's EEG showed a poorly persistent alpha background and extensive slow waves, mainly in the bilateral frontal and temporal lobes, suggesting cortical dysfunction.

HBO has been a traditional method of treating DEACMP in basic and clinical studies. Dexamethasone combined with $\mathrm{HBO}$ therapy has been reported to have better efficacy than HBO alone [13]. Myelin basic protein (MBP) levels in cerebrospinal fluid decreases significantly after treatment. Basic experiments have also confirmed that [14] dexamethasone can inhibit the immune response, preventing MBP degradation and DEACMP in the rat model. Furthermore, methylene blue was shown to be effective in a rat model of DEACMP [15]. Cognitive impairment often occurs in DEACMP $[16,17]$. Our case one showed difficulty in understanding, expression, memory, character, and spatial orientation. The mechanism may be related to the affection of nicotinic cholinergic system after DEACMP [18]. Recent studies have confirmed that acetylcholinesterase inhibitors such as donepezil or galanthamine could alleviate cognitive impairment associated with DEACMP. Interestingly, the latter seems to be better than the former $[19,20]$. Moreover, compared with $\mathrm{HBO}$ treatment alone, the combination of $\mathrm{N}$ butylphthalide and $\mathrm{HBO}$ for disease management may significantly improve the mini-mental state examination scores $[21,22]$.

To our knowledge, this is the first DEACMP report concerning bilateral cerebral peduncle anomalies captured on MRI. We further demonstrated with DTI that this symmetrical anomaly was due to the Wallerian degeneration of bilateral cerebral peduncles secondary to upstream axonal damage. This report may help further our understanding of DEACMP imaging.

Table 1 The differential diagnosis of bilateral cerebral peduncles abnormity

\begin{tabular}{|c|c|c|c|c|c|}
\hline Author & Date & Subject & Lesion & Etiology & Imaging \\
\hline Waragai [6] & 1994 & Human & Cerebral hemisphere & Stroke & $\mathrm{MRI}, \mathrm{T}_{2}$ image \\
\hline Zakaria [7] & 2006 & Human & $\begin{array}{l}\text { Basilar artery } \\
\text { posterior cerebral arteries }\end{array}$ & Stroke & DWI, MRA \\
\hline Tuor [8] & 2013 & Neonatal rat & Cerebral hemisphere & $\begin{array}{l}\text { Hypoxia } \\
\text { ischemia }\end{array}$ & $\begin{array}{l}\mathrm{MRl}, \mathrm{T}_{2} \text { image } \\
\mathrm{ADC}\end{array}$ \\
\hline
\end{tabular}




\section{Abbreviations}

ADC: Apparent dispersion coefficient; CO: Carbon monoxide; CT: Computed tomography; DTI: Diffusion tensor imaging; DEACMP: Delayed encephalopathy after acute carbon monoxide poisoning: EEG: Electroencephalography; GFAP: Glial fibrillary acidic protein; HBO: Hyperbaric oxygen; MBP: Myelin basic protein; MRI: Magnetic resonance imaging; MMSE: Mini-mental state examination

\section{Acknowledgements}

We would like to thank Xiao-Wei Wu, Lei Zhang, Ling Li and Bing Xu for imaging technical support

\section{Authors' contributions}

Writing - original draft: SYX. Writing - review and editing: CXL. Data curation: LYL, YS2. Conceptualization: YS1. All authors read and approved the final manuscript.

\section{Funding}

This study was supported by grants from China Cardiovascular Association (No. 2017-CCA-VG-048); Shanxi Provincial Health and Family Planning Commission (No. 2017033); Doctoral Fund of the First Hospital of Shanxi Medical University (No. YB161706, No. BS03201631); Shanxi Applied Basic Research Program (No. 201801D221426); Shenyang Committee of Science and Technology (No. 18-014-4-51). The patients' brain MRI examination, DTI testing, and manuscript writing were funded in this study. The funders had no role in the design of the study, collection, analysis, and interpretation of data.

\section{Availability of data and materials}

Not applicable.

\section{Ethics approval and consent to participate}

Since the images presented in the article are unidentifiable, the Ethics Committee of our institution waived the requirement for approval of this case study with medical records.

\section{Consent for publication}

Considering the both two patients had cognitive impairment, the informed written consent was obtained from the patients' next of kin for publication of this case report.

\section{Competing interests}

The authors declare that they have no competing interests.

\section{Author details}

${ }^{1}$ Department of Neurology, the First Hospital of Shanxi Medical University, Taiyuan, China. ${ }^{2}$ Department of Neurology, the First Affiliated Hospital of Shenzhen University, Health Science Center, Shenzhen Second People's Hospital, Shenzhen, China. ${ }^{3}$ Department of Radiology, Liaoning Jinqiu Hospital, Liaoning Provincial Geriatric Hospital, Shenyang, China. ${ }^{4}$ Department of Neurology and Neuroscience, Shenyang First People's Hospital, Shenyang Brain Hospital, Shenyang Brain Institute, Shenyang Medical College, Shenyang, China.

\section{Received: 23 June 2019 Accepted: 6 March 2020}

Published online: 17 March 2020

\section{References}

1. Chang CC, et al. Clinical significance of the pallidoreticular pathway in patients with carbon monoxide intoxication. Brain. 2011;134(Pt 12):3632-46.

2. Cree BAC, et al. Clemastine rescues myelination defects and promotes functional recovery in hypoxic brain injury. Brain. 2018;141(1):85-98.

3. Du X, et al. Utility of brain CT for predicting delayed encephalopathy after acute carbon monoxide poisoning. Exp Ther Med. 2019;17(4):2682-8.

4. Wang $X$, et al. MRI and clinical manifestations of delayed encephalopathy after carbon monoxide poisoning. Pak J Pharm Sci. 2016;29(6 Suppl):231720.

5. Sung YF, et al. Generalized chorea due to delayed encephalopathy after acute carbon monoxide intoxication. Ann Indian Acad Neurol. 2015;18(1): 108-10.

6. Waragai M, Watanabe $\mathrm{H}$, Iwabuchi S. The somatotopic localisation of the descending cortical tract in the cerebral peduncle: a study using MRI of changes following Wallerian degeneration in the cerebral peduncle after a supratentorial vascular lesion. Neuroradiology. 1994:36(5):402-4.

7. Zakaria T, Flaherty ML. Locked-in syndrome resulting from bilateral cerebral peduncle infarctions. Neurology. 2006;67(10):1889.

8. Tuor UI, et al. Magnetization transfer and diffusion imaging of acute axonal damage in the cerebral peduncle following hypoxia-ischemia in neonatal rats. Pediatr Res. 2013;73(3):325-31.

9. Kim JH, et al. Delayed encephalopathy of acute carbon monoxide intoxication: diffusivity of cerebral white matter lesions. AJNR Am J Neuroradiol. 2003;24(8):1592-7.

10. Funata $\mathrm{N}$, et al. Electron microscopic observations of experimental carbon monoxide encephalopathy in the acute phase. Acta Pathol Jpn. 1982;32(2): 219-29.

11. Di C, et al. Dynamic changes and clinical significance of serum S100B protein and glial fibrillary acidic protein in patients with delayed encephalopathy after acute carbon monoxide poisoning. Pak J Med Sci. 2018;34(4):945-9.

12. Sekiya K, et al. Carbon monoxide poisoning-induced delayed encephalopathy accompanies decreased microglial cell numbers: distinctive pathophysiological features from hypoxemia-induced brain damage. Brain Res. 2019;1710:22-32.

13. Xiang W, et al. Combined application of dexamethasone and hyperbaric oxygen therapy yields better efficacy for patients with delayed encephalopathy after acute carbon monoxide poisoning. Drug Des Devel Ther. 2017;11:513-9.

14. Li Q, et al. Dexamethasone therapy for preventing delayed encephalopathy after carbon monoxide poisoning. Biotech Histochem. 2015;90(8):561-7.

15. Zhao $\mathrm{N}$, et al. After treatment with methylene blue is effective against delayed encephalopathy after acute carbon monoxide poisoning. Basic Clin Pharmacol Toxicol. 2018;122(5):470-80.

16. Wang P, Zeng T, Chi ZF. Recovery of cognitive dysfunction in a case of delayed encephalopathy of carbon monoxide poisoning after treatment with donepezil hydrochloride. Neurol India. 2009:57(4):481-2.

17. Song IU, et al. Improvement of cognitive impairment following delayed CO encephalopathy. Can J Neurol Sci. 2011:38(6):934-5.

18. Ochi $\mathrm{S}$, et al. The nicotinic cholinergic system is affected in rats with delayed carbon monoxide encephalopathy. Neurosci Lett. 2014;569:33-7.

19. Tseng WT, et al. Effects of donepezil on cognition and global functioning in patients with delayed encephalopathy after carbon monoxide poisoning - a case series. Psychiatry Clin Neurosci. 2019;73:348.

20. Yanagiha K, Ishii K, Tamaoka A. Acetylcholinesterase inhibitor treatment alleviated cognitive impairment caused by delayed encephalopathy due to carbon monoxide poisoning: two case reports and a review of the literature. Medicine (Baltimore). 2017:96(8):e6125.

21. Wang $\mathrm{H}$, et al. Combination of butylphthalide with umbilical mesenchymal stem cells for the treatment of delayed encephalopathy after carbon monoxide poisoning. Medicine (Baltimore). 2016;95(49):e5412.

22. Xiang W, et al. Efficacy of N-Butylphthalide and hyperbaric oxygen therapy on cognitive dysfunction in patients with delayed encephalopathy after acute carbon monoxide poisoning. Med Sci Monit. 2017:23:1501-6.

\section{Publisher's Note}

Springer Nature remains neutral with regard to jurisdictional claims in published maps and institutional affiliations.

\section{Ready to submit your research? Choose BMC and benefit from:}

- fast, convenient online submission

- thorough peer review by experienced researchers in your field

- rapid publication on acceptance

- support for research data, including large and complex data types

- gold Open Access which fosters wider collaboration and increased citations

- maximum visibility for your research: over $100 \mathrm{M}$ website views per year

At $\mathrm{BMC}$, research is always in progress.

Learn more biomedcentral.com/submissions 